\title{
BMJ Open Comparative efficacy of interventions for reducing injection and sexual risk behaviours to prevent HIV in injection drug users: protocol for Bayesian network meta-analysis
}

Junjie Lang, Lairun Jin, Yingshui Yao

To cite: Lang J, Jin L, Yao Y. Comparative efficacy of interventions for reducing injection and sexual risk behaviours to prevent HIV in injection drug users: protocol for Bayesian network meta-analysis. BMJ Open 2019;9:e022811. doi:10.1136/ bmjopen-2018-022811

- Prepublication history for this paper is available online. To view these files, please visit the journal online (http://dx.doi org/10.1136/bmjopen-2018022811).

Received 7 March 2018 Revised 12 November 2018 Accepted 13 November 2018
A) Check for updates

(C) Author(s) (or their employer(s)) 2019. Re-use permitted under CC BY-NC. No commercial re-use. See rights and permissions. Published by BMJ.

School of Public Health, Wannan Medical College, Wuhu, China

Correspondence to

Dr Yingshui Yao;

yingshuiya0@163.com

\section{ABSTRACT}

Introduction Drug users are more vulnerable to AIDS than the general population. While several interventions are effective for addressing HIV in injection drug users, no meta-analysis has yet been performed to compare interventions and determine the relative benefits of each. We intend to conduct a Bayesian network meta-analysis to compare all available interventions evaluated by a randomised controlled trial for reducing injection and risky sexual behaviours for the prevention of HIV in injection drug users.

Methods and analysis Studies will be retrieved by searching the following databases: MEDLINE, Embase, PsycINFO and Cochrane Central Register of Controlled Trials. The search will be performed between May and July 2018 for the literature published between 1980 and May 2018. Two authors will extract data independently. Primary outcome measures will be injection risk behaviour and HIV risk behaviour. HIV seroconversion, confirmed using an antibody test, will be the secondary outcome. Bayesian network meta-analyses will be conducted using the Markov Chains Monte Carlo method. The Cochrane revised tool, Risk of Bias, will be used to assess the risk of bias. Grading of Recommendations Assessment, Development and Evaluation will be used to assess evidence quality. Ethics and dissemination The results of this study will be disseminated at professional conferences and via publications in peer-reviewed journals. This study will not include any confidential personal data or data on human trials; therefore, ethical approval is not required.

PROSPERO registration number CRD42018086999.

\section{INTRODUCTION}

Injecting drug users (IDUs) are known to be at higher risk of HIV infection than the general population. Data from the United Nations Office on Drugs and Crime (UNODC) indicate that the number of people who inject drugs worldwide is approximately 12.7 million. ${ }^{1}$ The 2018 UNODC/ WHO/The Joint United Nations Programme on HIV/AIDS (UNAIDS)/World Bank global estimate of the number of IDUs and

\section{Strengths and limitations of this study}

- This meta-analysis will conduct a comprehensive comparison of interventions for reducing injection and sexual risk behaviours to prevent HIV in injection drug users.

- This protocol is written in strict accordance with the Preferred Reporting Items for Systematic Reviews and Meta-Analyses Protocols.

- This meta-analysis will be limited to studies which are published in English language and have been peer reviewed.

- Given this meta-analysis will only include randomised controlled trials, there is a possibility that the study participant population will not be representative of the overall population.

- We only focus on persons who inject drugs and interventions defined by WHO, United Nations Office on Drugs and Crime and United Nations Programme on HIV/AIDS (nor all interventions).

are living with HIV was 1.7 million (range: 0.9-4.8 million), corresponding to an average prevalence of HIV among IDUs of $13.1 \%{ }^{2}$ Furthermore, based on data published by UNAIDS, injecting drugs users accounted for $51 \%$ of people with HIV infections in eastern Europe and central Asia, and 13\% of new HIV infections in Asia and the Pacific, in 2014. ${ }^{3}$

HIV is a major contributor to the disease burden attributable to drug use globally. ${ }^{4}$ Effective interventions are necessary to address HIV in injection drug users. There is a comprehensive package of nine interventions, endorsed by UNAIDS, UNODC and $\mathrm{WHO}$, for the prevention, treatment and care of HIV in IDUs, which includes: needle and syringe programmes (NSPs); opioid substitution therapy (OST); antiretroviral therapy; and targeted information, education and communication (IEC) (among other measures) ${ }^{1}$ 
There have been several systematic reviews and meta-analyses of HIV interventions in IDUs. ${ }^{5-9}$ These studies have confirmed the efficacy of interventions such as NSPs,${ }^{59}$ psychosocial interventions and IEC ${ }^{6-8}$; however, none of the meta-analyses evaluated the effects of all of these interventions or compared the relative benefits of each; therefore, information regarding whether distinct types of intervention have comparable efficacy and are equally appropriate for different populations of injection drug users are lacking.

\section{Objectives}

In this study, we aim to compare the efficacy of all available interventions for reducing injection and sexual risk behaviours to prevent HIV in injection drug users.

\section{METHODS AND ANALYSIS}

A network meta-analysis can combine direct and indirect evidence to provide more precise and accurate (thus both internally and externally valid) effect estimates. ${ }^{10}$ Moreover, based on effective statistical inference methods, it allows ranking of investigated interventions to determine which among them is the most and least effective. ${ }^{11}$

This protocol follows the Preferred Reporting Items for Systematic Reviews and Meta-Analyses Protocols (PRISMA-P) ${ }^{12}$ It has also been registered in the International Prospective Register of Systematic Reviews (trial registration number: CRD42018086999).

\section{Eligibility criteria for reports focused on}

Types of participant

People who inject opiates, cocaine, cannabis and amphetamines (including 'ecstasy') will be included. People who primarily misuse alcohol will be excluded.

\section{Interventions}

Interventions which are defined by WHO, UNODC and UNAIDS will be included ${ }^{1}$ :

- NSPs.

- OST and other evidence-based drug dependence treatment programmes.

- HIV testing and counselling (HTC).

- Antiretroviral therapy (ART).

- Prevention and treatment of sexually transmitted infections.

- Condom programmes.

- Targeted IEC for people who inject drugs.

\section{Comparators}

Placebo-controlled or no intervention. Studies which compare two different interventions within the same investigation will also be accepted.

\section{Outcomes}

Injection risk behaviour, sexual risk behaviour, or HIV seroconversion.
Study designs and publication types

Randomised controlled trials and peer-reviewed publications.

Setting

Any healthcare setting.

\section{Language and time frame}

We will include studies which are published in English and published from 1980 to May 2018.

\section{Information sources and search strategy}

We will search the following databases: MEDLINE, Embase, PsycINFO and the Cochrane Central Register of Controlled Trials. The search will be performed between May and July 2018. The search strategy shown below was adapted from a previous review, ${ }^{13}$ and improved by conferring with experts in a related field. The search strategies for other databases will be adjusted according to their specific requirements. We will also carry out manual searches of the reference lists of other review articles on related subjects, to retrieve additional studies not identified by our original search. The following search terms will be used:

1. 'Drug users' OR 'drug use' $O R$ 'drug abuse' $O R$ 'drug abuser' OR 'drug abusers' OR 'drug addict*' OR 'substance abuse' OR 'substance dependence' OR 'drug dependence' OR 'drug dependency' OR 'IDU' OR 'IDUs' OR 'injecting drug' OR 'intravenous drug' OR 'intravenous substance' OR 'injecting substance' OR exp substance abuse, intravenous/

2. 'HIV' OR 'AIDS' OR 'acquired immunodeficiency syndrome' OR 'Acquired Immunodeficiency Syndrome Virus' OR 'AIDS Virus' OR 'AIDS Viruses' OR 'Immunologic Deficiency Syndrome, Acquired' OR 'Acquired Immune Deficiency Syndrome’ OR exp HIV/ OR exp HIV Infections/

3. \#1 AND \#2

4. *Randomised Controlled Trial/OR (Randomised Controlled Trial).pt OR *Random Allocation/.

5. (Randomised OR randomised OR (random* adj (assigned OR allocated OR assignment OR allocation))). ab,ti.

6. \#4 OR \#5

7. \#3 AND \#6

\section{Study selection}

We will import the search results into EndNote (data management software). After removing duplicate articles, the first two authors will independently read the titles and abstracts to select eligible articles according to the inclusion criteria. Then we will obtain the full texts of all articles which appear to meet the inclusion criteria or where there is any uncertainty. The first two authors will conduct full-text reviews independently to confirm the eligibility of these articles. Cohen's Kappa ( $\kappa$ ) (calculated by R software 3.44) will be used to measure the chance-corrected agreement between the two authors. Any discrepancies will be resolved by discussion with a third author and 
the reasons for excluding articles at full report will be recorded.

\section{Data collection process}

The first two authors will independently use Excel 2016 software to abstract the following information from the articles collected as described above:

1. Study characteristics (first author, journal, year, country, sample size and so on).

2. Participant characteristics (age, sex, manner of drug use, type of drug, the incidence rate of injection risk behaviours or sexual risk behaviours at baseline and so on).

3. Intervention characteristics (type, treatment dose, and duration and so on).

4. Control characteristics.

Any disagreements will be resolved by discussion with the third author and we will contact the original authors of studies to resolve any uncertainties if necessary.

\section{Outcome measures}

Our primary outcome measures will be injection risk behaviours and HIV risk behaviours. HIV seroconversion confirmed by an antibody test will be the secondary outcome, if available. The efficacy will be based on the difference in injection risk behaviours and HIV risk behaviours between the intervention and comparator on the completion of intervention. HIV risk behaviours include sex types (vaginal or anal), frequency of condom protected sex and whether engaging in sex with other partners concurrently. Injection risk behaviour will be defined as having shared syringes, containers, filters or water to inject drugs in the previous month and backloading/frontloading. We will review all the acquired full texts to check the relative scale used by each study, for example, the HIV Risk-Taking Behaviour Scale, ${ }^{14}{ }^{15}$ to assess the above indicators.

\section{Risk of bias in individual studies}

We will use the Cochrane revised tool, Risk of Bias (RoB $\mathrm{V} .2 .0),{ }^{16}$ to determine the risk of bias. The RoB tool contains five key domains: (1) randomisation process; (2) deviations from intended interventions; (3) missing outcome data; (4) measurement of the outcome; and (5) selective reporting. The risk of bias will be classified into three types for each domain: high, low or some concerns. Subsequently, we will arrive at an overall risk of bias, based on judgements from the five domains. A summary of risk of bias of all the domains will be provided for each trial. The first two authors will perform all assessments independently of each other. Any disagreements will be resolved by discussion with a third author.

\section{Data synthesis}

We will use Stata software (13.0; Stata Corporation) to conduct a traditional pairwise meta-analysis. If more than five studies are included, we will use the random effects model to combine the data. Otherwise, we will use a fixed effect model, because the random effects model may be imprecise in this situation. ${ }^{17}$ Dichotomous data will be evaluated using the risk ratio (RR) with $95 \%$ CI, while continuous outcomes will be expressed as standardised mean differences and 95\% CI. Heterogeneity will be quantified using the $\mathrm{I}^{2}$ statistic. If $\mathrm{I}^{2}>50 \%$, which indicates the presence of substantial heterogeneity, ${ }^{18}$ we will consider subgrouping the intervention by study setting (receiving formal treatment or not), gender-specific (single-gender or mixed gender), HIV testing (reported or not), methodological quality of the study, drug types or geographical area.

When pairwise meta-analysis is completed, we will perform a network meta-analysis using WinBUGS 1.43 software. The Markov Chains Monte Carlo method will be used for Bayesian analysis. When we run the WinBUGS programme, we will set it to perform 100000 simulations, and the first 10000 simulations will be discarded as burnin. Convergence of the model will be assessed by trace and Gelman-Rubin-Brooks plots. ${ }^{19}$

We will use both random-effects and fixed-effects models for the network meta-analysis. Then we will select the appropriate model on the basis of the deviance information criterion (DIC); the model with the lower DIC will be preferred (a difference $>3$ will be considered significant). ${ }^{20}$ The node-splitting method will be used to statistically assess the consistency between direct and indirect evidence. ${ }^{21}$

We will examine the assumptions of transitivity (similarity in the distribution of potential effect modifiers across the different pairwise comparisons) on the account of clinical and methodological characteristics; notably, there is no universal statistical method to analyse these effect modifiers. ${ }^{22}{ }^{23}$ We plan to investigate similarity based on factors including participant characteristics, experimental design, study quality and risk of bias, among others.

Furthermore, publication bias will be assessed using comparison-adjusted funnel plots. All the figures, including forest plots for each intervention, network plots and comparison-adjusted funnel plots, will be produced using the 'Network Graphs' package in STATA.

We will performed sensitivity analysis to address whether the combined estimates of the interventions are dominated by one or several studies, especially those with a high risk of bias. Then we will exclude the trials to test the robustness of our study result. Second, we will test whether the imputation of the missing values affects the result of the meta-analysis.

\section{Confidence in cumulative evidence}

We will evaluate the quality of evidence for all outcomes according to the Grading of Recommendations Assessment, Development and Evaluation. Based on the domains of methodology quality, consistency, directness, precision effect estimates and publication bias, we will rank the overall strength of evidence as high, moderate, low or very low. ${ }^{24}$ 
Patient and public involvement

Patients will not be involved.

\section{ETHICS AND DISSEMINATION}

This Bayesian network meta-analysis will include no confidential personal data and no data on human trials. Consequently, ethical approval is not required. The procedures used for this study will be reported in accordance with the Preferred Reporting Items for Systematic Reviews and Meta-Analysis (PRISMA) extension statement for network meta-analyses of healthcare interventions. ${ }^{25}$ The final results will be disseminated at professional conferences and through publications in peer-reviewed journals.

Contributors $\mathrm{JL}$ contributed to the conception and design of this study protocol and is the guarantor of the protocol. YY developed the search strategy. LJ will provide advice on data analysis and presentation of study results. All authors approved the final version of the manuscript.

Funding Anhui Provincial Department of Education upgrade quality project Master Studio(2014msgzs151); Key Projects for Academic Support of Top-level Talents in Universities(gxbjZD2016073).

Competing interests None declared.

Patient consent for publication Not required.

Provenance and peer review Not commissioned; externally peer reviewed.

Open access This is an open access article distributed in accordance with the Creative Commons Attribution Non Commercial (CC BY-NC 4.0) license, which permits others to distribute, remix, adapt, build upon this work non-commercially, and license their derivative works on different terms, provided the original work is properly cited, appropriate credit is given, any changes made indicated, and the use is non-commercial. See: http://creativecommons.org/licenses/by-nc/4.0/.

\section{REFERENCES}

1. World Health Organization, United Nations Office on Drugs and Crime, and Joint United Nations Programme on HIV/AIDS. WHO, UNODC, UNAIDS technical guide for countries to set targets for universal access to HIV prevention, treatment and care for injecting drug users. Geneva: World Health Organization, 2012. http://apps. who.int/iris/bitstream/10665/77969/1/9789241504379_eng.pdf?ua=1 (Accessed 24 Dec 2017).

2. United Nations Office on Drugs and Crime. World drug report, 2018. http://www.unodc.org/wdr2018/prelaunch/WDR18_Booklet_1_ EXSUM.pdf (Accessed 25 Aug 2018).

3. UNAIDS. Global AIDS Update. 2016 http://www.who.int/hiv/pub/arv/ global-aids-update-2016-pub/en/ (Accessed 25 Dec 2017).

4. Degenhardt L, Hall W, Lynskey M, et al. Illicit drug use. In: Ezzati M, Lopez AD, Rodgers A, eds. Comparative quantification of health risks: global and regional burden of disease attributable to selected major risk factors. 2nd edn. Geneva: World Health Organization, 2004:1109-76.
5. Jones L, Pickering L, Sumnall $\mathrm{H}$, et al. Optimal provision of needle and syringe programmes for injecting drug users: A systematic review. Int J Drug Policy 2010;21:335-42.

6. Meader N, Li R, Des Jarlais DC, et al. Psychosocial interventions for reducing injection and sexual risk behaviour for preventing HIV in drug users. Cochrane Database Syst Rev 2010:CD007192.

7. Semaan S, Des Jarlais DC, Sogolow E, et al. A meta-analysis of the effect of HIV prevention interventions on the sex behaviors of drug users in the United States. J Acquir Immune Defic Syndr 2002;30(Suppl 1):S73-S93.

8. Prendergast ML, Urada D, Podus D. Meta-analysis of HIV riskreduction interventions within drug abuse treatment programs. $J$ Consult Clin Psychol 2001;69:389-405.

9. Fernandes RM, Cary M, Duarte G, et al. Effectiveness of needle and syringe Programmes in people who inject drugs - An overview of systematic reviews. BMC Public Health 2017;17:309.

10. Biondi-Zoccai G, Abbate A, Benedetto U, et al. Network metaanalysis for evidence synthesis: what is it and why is it posed to dominate cardiovascular decision making? Int $J$ Cardiol 2015;182:309-14.

11. Greco T, Landoni G, Biondi-Zoccai G, et al. A Bayesian network meta-analysis for binary outcome: how to do it. Stat Methods Med Res 2016;25:1757-73.

12. Shamseer L, Moher D, Clarke M, et al. Preferred reporting items for systematic review and meta-analysis protocols (PRISMA-P) 2015: elaboration and explanation. BMJ 2015;350:g7647.

13. Mathers BM, Degenhardt L, Ali $H$, et al. HIV prevention, treatment, and care services for people who inject drugs: a systematic review of global, regional, and national coverage. Lancet 2010;375:1014-28.

14. Darke S, Hall W, Heather N, et al. The reliability and validity of a scale to measure HIV risk-taking behaviour among intravenous drug users. AIDS 1991;5:181-6.

15. Ward J, Darke S, Hall W. The HIV Risk-taking behaviour scale (HRBS) manual, 1990.

16. Higgins JPT, Sterne JAC, Savović J, et al. A revised tool for assessing risk of bias in randomized trials. In: Chandler J, McKenzie $\mathrm{J} \mathrm{B}$, eds. Cochrane methods cochrane database of systematic reviews, 2016.

17. Higgins JP, Thompson SG, Spiegelhalter DJ. A re-evaluation of random-effects meta-analysis. J R Stat Soc Ser A Stat Soc 2009;172:137-59.

18. Higgins JP, Thompson SG, Deeks JJ, et al. Measuring inconsistency in meta-analyses. BMJ 2003;327:557-60.

19. Gelman A, Rubin DB. Inference from iterative simulation using multiple sequences. Statistical Science 1992;7:457-72.

20. Spiegelhalter DJ, Best NG, Carlin BP, et al. Bayesian measures of model complexity and fit. Journal of the Royal Statistical Society: Series B 2002;64:583-639.

21. Dias S, Welton NJ, Caldwell DM, et al. Checking consistency in mixed treatment comparison meta-analysis. Stat Med 2010;29:932-44.

22. $\mathrm{Kim} \mathrm{H}$, Gurrin L, Ademi Z, et al. Overview of methods for comparing the efficacies of drugs in the absence of head-to-head clinical trial data. Br J Clin Pharmacol 2014;77:116-21.

23. Jansen JP, Naci H. Is network meta-analysis as valid as standard pairwise meta-analysis? It all depends on the distribution of effect modifiers. BMC Med 2013;11:159.

24. Schunemann H, Brozek J, Guyatt G, et al. GRADE handbook for grading quality of evidence and strength of recommendation, 2013. http:// gdt. guidelinedevelopment. org/ app/ (Assessed 22 Dec 2017).

25. Hutton B, Salanti G, Caldwell DM, et al. The PRISMA extension statement for reporting of systematic reviews incorporating network meta-analyses of health care interventions: checklist and explanations. Ann Intern Med 2015;162:777-84. 